\title{
Topological solutions in dual formulation of Yang-Mills theories
}

\author{
A.S. Gorsky \\ Institute of Theoretical and Experimental Physics, Moscow \\ E-mail: gorsky@itep.ru \\ Valentin I Zakharov* \\ Max Planck Institut fuer Physik, Munich \\ E-mail: xxz@mppmu.mpg.de
}

\begin{abstract}
We review briefly the emerging picture of the confinement-deconfinement phase transition as transition from four-dimensional to three-dimensional non-perturbative physics. In particular we consider the so called magnetic component of the Yang-Mills plasma. We comment on the recent lattice observation that the thermal monopoles in $\mathrm{SU}(2)$ gauge theories appear to possess both magnetic and electric color fields. We attempt to fit the observation into the general scheme of Yang-Mills defects developed earlier within the Sakai-Sugimoto model. The geometric features of the defects in the Yang-Mills vacuum reflect the topology of extra dimensions in this model. We favor the classification which assumes that the color magnetic and electric of the monopoles are not parallel to each other. The prediction can be checked on the lattice.
\end{abstract}

International Workshop on QCD Green's Functions, Confinement, and Phenomenology - QCD-TNT09 September 07 - 112009

ECT Trento, Italy

\footnotetext{
*Speaker.
} 


\section{Introduction}

For many years the quark-gluon plasma was thought of as a weakly interacting gas, with Debye screening of color charges. After the RHIC experiments, resulted in discovery of the 'strongly interacting plasma', the precise nature of the confinement-deconfinement phase transition became much more obscure.

Probably, the physics of confinement and deconfinement could be best understood within the models which attribute the confinement to the stochastic component of vacuum associated with defects of lower dimensions. The models were originally formulated within lattice studies, or in Euclidean space, for review and references see, e.g., [1]. The most popular candidates are 1d defects, or trajectories which are called monopole trajectories or $2 \mathrm{~d}$ defects (surfaces) called center vortices. The stochasticity is manifested as percolation of the trajectories or surfaces in $4 \mathrm{~d}$ space. For many reasons, the idea was not immediately picked up by the continuum-theory community. In particular, the definitions of the defects are originally given in specific lattice language which has no direct continuum-theory translation ${ }^{1}$.

For us, it is most important now that the lattice studies suggested in fact another interpretation of the deconfinement phase transition as a change from four to three dimensions:

$$
\text { confinement } \rightarrow \text { deconfinement } \equiv 4 d \rightarrow 3 d,
$$

where the change of the dimensionality is understood in pure geometrical terms: the defects become time oriented and percolate in $3 \mathrm{~d}$ rather than in $4 \mathrm{~d}$. Moreover, the surviving $3 \mathrm{~d}$ stochasticity explains naturally the persistence of the area law for the spatial Wilson in the deconfining phase.

If one starts with the standard, field-theoretic formulation of QCD the very existence of the lower dimensional vacuum defects remains mysterious. Things are different within the dual. or string models of QCD where lower dimensional defects are common. Moreover, within the holographic models of QCD the deconfining phase transition in terms of the defects looks generically similar [3] to the picture observed first in lattice studies, see above. In some cases, the holographic models and lattice data agree with each other to amusingly fine details [4] ${ }^{2}$.

In more detail, we will rely on the Sakai-Sugimoto holographic model [6] which is most successful in describing low-energy QCD. The metrics of this model is formulated in terms of the ordinary $4 \mathrm{~d}$ space $\left(t, x^{i}, i=1,2,3\right)$, extra fifth dimension $u$ with horizon at $u=u_{\Lambda}$, one more extra compact dimension $x^{4}$ and compact sphere $\Omega_{4}$ :

$$
\begin{array}{r}
d s^{2}=\left(\frac{u}{R_{0}}\right)^{3 / 2}\left(-d t^{2}+\delta_{i j} d x^{i} d x^{j}+f(u) d x_{4}^{2}\right)+\left(\frac{u}{R_{0}}\right)^{-3 / 2}\left(\frac{d u^{2}}{f(u)}+u^{2} d \Omega_{4}^{2}\right) \\
f(u)=1-\left(\frac{u_{\Lambda}}{u}\right)^{3} .
\end{array}
$$

Because of the compactness of the $x^{4}$-coordinate there exist topological defects wrapped around the $x^{4}$-circle. These defects have non-trivial $\theta$-angle dependence. Note that the metric (1.1) in

\footnotetext{
${ }^{1}$ For the purpose of the present note we oversimplify the story greatly. There were in fact numerous attempts to interpret the lattice defects in the continuum-theory language, for review and references see, e.g. [2]

${ }^{2}$ Remarkably enough, the properties of the lattice vortices are also close to the properties of string-like solutions of the non-Abelian SUSY theories, see [5] and references therein.
} 
coordinates $\left(x^{4}, u\right)$ has a cigar=like form so that the radius of the $x^{4}$ compact dimension tends to zero at the horizon $u=u_{\Lambda}$. Which means that the defects wrapped around this dimension have tension vanishing at the horizon ${ }^{3}$.

At finite temperature $\mathrm{T}$ it is convenient to use Euclidean space, with a compact time direction $\tau, 0<\tau<1 / T$. Thus, at $T \neq 0$ there are two compact coordinates, $x^{4}$ and $\tau$. The deconfining phase transition is then understood as change of geometries in two-dimensional $\left(x^{4}, u\right)$ and $(\tau, u)$ subspaces. Namely, at $T>T_{c}$ the cigar-shape geometry sets in the $\tau, u$ coordinates while in the $\left(x^{4}, u\right)$ coordinates the geometry becomes cylinder-like. As a result, defects wrapped in (Euclidean) time direction become tensionless and could condense.

As is argued in $[8,3]$ this is a generic phenomenon within the dual model: below $T_{c}$ defects condensed in the vacuum have nontrivial $\theta$-angle dependencies while at $T>T_{c}$ it is the defects wrapped on the compact time direction which are tensionless.

This observation implies that at $T>T_{c}$ generically defects become time-oriented since in this case the associated tension tends to zero. Thus, geometry of the extra dimensions is manifested in geometrical structure of the defects in ordinary $4 \mathrm{~d}$ Euclidean space, exactly in the same way as was observed first on the lattice (see above).

Of course, this is only a generic picture and details, sometimes crucial ones depend on the particular defect considered. So far discussion $[4,3,2]$ was concentrated mostly on D2 branes, or vortices. Some other defects were listed in [3]. It happened so, however, that the most recent lattice data [9] refers to the so called wrapped monopoles, see [10] and references therein. This type of defects has not been explicitly discussed in [3] and we address the issue in these notes.

\section{Wrapped, or thermal monopoles}

Monopoles on the lattice are defined usually in specific lattice (or algorithmic) language using a particular projection of the original Abelian fields onto an Abelian subclass of the fields. The most successful is the so called Maximal Abelian Projection, for review see [12]. The words 'most successful' are to be understood as the statement that these monopoles exhibit many explicitly $\mathrm{SU}(2)$ invariant properties, like non-Abelian action, length of trajectories in physical units and so on, for review see [2]. Thus, one can conclude that through the use of the projection, one detects gauge-invariant objects.

The monopole trajectories are closed, by definition. In the continuum-theory language the closeness corresponds to a charged particle, or a complex scalar field, see, e.g., [13]. At finite temperature there appear new types of monopole trajectories, the so called wrapped trajectories. In more detail, finite temperature $T$ assumes periodicity in the (Euclidean) time direction with period $1 / T$. Thus, if a monopole trajectory reaches the boundary in the time direction it is automatically continued to the opposite boundary. For each of such trajectories one can introduce the wrapping number, $s$ which counts the number of times the trajectory hits the boundary before becoming a close trajectory.

\footnotetext{
${ }^{3}$ This is true in the classical limit. It remains unclear, therefore, in this limit whether the defects remain physical. As is argued in particular in [2, 4, 3] analysis of the lattice data suggests strongly that the defects with classically vanishing tension become percolating vacuum structure, with observed density scaled in physical units which implies that they are physical indeed.
} 
It was argued in Ref. [10] that the average wrapping number (measured in the Euclidean space) is directly related to the density $\rho(T)$ of the corresponding particles in the actual plasma (in Minkowskian space):

$$
\rho(T)=n_{w r}=<|s|>/ V_{3 d},
$$

where $V_{3 d}$ is the three-dimensional volume of the lattice. Eq. (2.1) is true, in particular, for free particles. If it is also true indeed for the wrapped, or thermal monopoles the density (2.1) should satisfy the scaling law

$$
n_{w r} \sim \Lambda_{Q C D}^{3},
$$

i.e. to be independent of the lattice spacing $a$ provided that the connection between $a$ and $\Lambda_{Q C D}$ is fixed by the standard renormgroup equations.

In practice, the scaling laws like (2.2) represent a strong test of theory. Lattice measurements [11] did demonstrate the validity of the prediction (2.2). Which is a strong argument in favor of the hypothesis that the wrapped monopoles correspond indeed to physical objects.

Most recently a further effort was made [9] to check this hypothesis through measurements of excess of the non-Abelian action associated with the wrapped monopoles. The measurements did demonstrate that the wrapped monopoles are associated with the extra action (for similar measurements for condensed monopoles at $\mathrm{T}=0$ see [14] and references therein).

Moreover, the wrapped monopole trajectories are preferably time-oriented and beginning with $T \approx 1.7 T_{c}$ become practically time-aligned [15]. Thus, one can distinguish between electric and magnetic color fields associated with the wrapped monopoles. The magnetic and electric fields are defined in terms of the unit vector $n_{\mu}$ looking in the direction of the monopole trajectory:

$$
H_{\mu}^{a} \equiv \tilde{G}_{\mu \nu}^{a} n_{\mu}, E_{\mu}^{a} \equiv G_{\mu \nu}^{a} n_{\mu},
$$

where $G_{\mu \nu}^{a}$ is the color field associated with the monopole (a is the color index, a=1,2,3, in the $\mathrm{SU}(2)$ case considered).

A remarkable finding [9] is that the magnetic and electric fields of the monopoles are equal to each other:

$$
\left|\mathbf{H}_{\text {mon }}\right|^{2}=\left|\mathbf{E}_{\text {mon }}\right|^{2} .
$$

Note that the mutual orientation of the vectors $\mathbf{H}, \mathbf{E}$ has not been measured so far. As for the absolute value of the fields, they are measured on the plaquettes closest to the monopole trajectory and exhibit scaling law similar to the $\mathrm{T}=0$ case:

$$
\left|\mathbf{H}_{m o n}\right|^{2}=\left|\mathbf{E}_{m o n}\right|^{2} \sim a^{-4},
$$

where $a$ is the lattice spacing. Assuming the fields being spherically symmetric the observation (2.5) implies

$$
\left|\mathbf{H}_{\text {mon }}\right|^{2}=\left|\mathbf{E}_{\text {mon }}\right|^{2} \sim \frac{1}{r^{4}},
$$

where $r$ is the distance to the center of the monopole.

The observations (2.4) and (2.5) not only dramatically confirm the physical nature of the wrapped monopoles but also make actual interpretation of these, newly emerging physical objects. 


\section{Thermal monopoles as dyons}

For simplicity of discussion we will assume that the thermal monopoles are static, i.e. the wrapped trajectories are time oriented. The issue not yet resolved by the measurements is the mutual orientation of the magnetic and electric color fields associated with the monopoles.

The possibility favored in discussion of the data in Ref. [9] is that the thermal monopoles are like dyons, i.e.

$$
\mathbf{H}^{a} \| \mathbf{E}^{a},\left(\mathbf{H}^{a}(r)\right)^{2}=\left(\mathbf{E}^{a}(r)\right)^{2} \sim r^{-4}
$$

The identification of the thermal monopoles with real dyonic particles leads, however, to some difficulties.

Indeed, if the monopole is a scalar particle then it may not have both electric and magnetic charges. Another way to express this point is to observe that a scalar particle has only one invariant, that is its mass. If there is an electric or magnetic field associated with it then the integral from the field can be included into the renormalization of the mass:

$$
\delta m \sim \int d^{3} x\left(\mathbf{E}^{2}+\mathbf{H}^{2}\right)
$$

However, if there exists another (P-odd) quantity constructed on the fields,

$$
\delta m_{5} \sim \int d^{3} x(\mathbf{E} \cdot \mathbf{H}) \neq 0,
$$

it cannot be associated with any invariant in the field theoretic description of the scalar particle.

If the monopoles are described by a spinor field $\psi$, then one can introduce both P-even and P-odd masses,

$$
\delta L=\delta m \bar{\psi} \psi+\delta m_{5} \bar{\psi} \gamma_{5} \psi
$$

and accommodate the both invariants (3.1 and (3.2). This hypothesis still have other difficulties. First, one would expect that the color fields associated with the spin 1/2 particles are dipole like,

$$
\mathbf{H}^{a} \| \mathbf{E}^{a},\left(H^{a}\right)^{2}(r)=\left(E^{a}\right)^{2} \sim r^{-6},
$$

and there is no indication of such a behavior in the lattice data [9].

Moreover, scalar and spinor particles in the random-walk, or polymer approach have different types of trajectories, see, e.g., [13]. The monopole trajectories were studied in great detail at temperature $T=0[16]$ and there is no sign whatsoever for a spinor-particle type of trajectories. The only change in geometry of the trajectories around $T=T_{c}$ is their time-orientation which universal for all defects, see Introduction, and is not related to the spin of the particles.

To summarize, it would be difficult to reconcile an ordinary-particle interpretation of the monopoles with basic theoretical expectations ${ }^{4}$. In geometrical terms, color fields of ordinary particles are spherically symmetric. If the monopoles belong, say, to surfaces their field may well be not spherically symmetry but, for example, confined to the surface. Moreover, this seems to be true for monopoles at temperature $T=0$, see, in particular, [2, 17, 5, 4] and references therein. Thus, it is natural to turn to interpretation of the thermal monopoles as elements of geometrically more complicated defects.

\footnotetext{
${ }^{4} \mathrm{We}$ assume at this point that, indeed, the thermal monopoles in the Euclidean space correspond to real particles in the Minkowski space [10]. For pure Euclidean defects self-dual non-Abelian fields are allowed.
} 


\section{Brane constructions}

Trivially, the monopoles look as D0 branes, or lines wrapped around the compact time direction. The fact that they become time oriented at temperatures not much exceeding $T_{c}$ finds its explanation since such defects have vanishing tension, see the Introduction. In the large $N_{c}$ limit the time orientation would set in much sharper than in case of $S U(2)$ theory studied in the lattice simulations so far. This is one more confirmation of the geometrical picture inherent to the Sakai-Sugimoto model known from other examples ${ }^{5}$.

The D0 branes could be a part of a more complicated construction. In particular, the fundamental, or electric strings can end on the D0. Such an assumption would explain that the color electric field is associated with the thermal monopoles.

Another point is that at $T=0$ the monopoles belong to magnetic D2 branes. These D2 branes are wrapped around the compact $x^{4}$ dimension and look as strings, or surfaces in the Euclidean $4 \mathrm{~d}$ space. This picture at $\mathrm{T}=0$ is strongly supported by various theoretical models and lattice data, see $[2,4,5,18]$. It is quite probable that also at $T>T_{c}$ the thermal monopoles are still a part of magnetic strings and this would explain naturally that there is magnetic field associated with thermal monopoles.

Moreover, the whole construction could be stable only because of the large angular momentum, in the spirit of the Myers phenomenon (for review see [19]). Then electric and magnetic fields are to be orthogonal to each other,

$$
\mathbf{E}^{a} \cdot \mathbf{H}^{a}=0 .
$$

It would be very interesting to check the mutual orientation of the magnetic and electric color fields associated with the thermal monopoles.

\section{Localization of classical solutions}

It might worth emphasizing that according to the lattice data the extra action associated with the thermal monopoles is ultraviolet divergent:

$$
\Delta S_{\text {monopole }} \sim L_{\text {trajectory }} / a
$$

where $S_{\text {monopole }}$ is the full non-Abelian action, $L$ is the length of trajectory and $a$ is the lattice spacing which goes to zero, $a \rightarrow 0$ in the continuum limit. On the other hand, theoretically one would rather expect

$$
S_{\text {monopole }} \sim L \cdot \Lambda_{Q C D} .
$$

arguing that the monopoles should be an infrared phenomenon, not seen in the ultraviolet.

Ar first sight, Eqs (5.1) and (5.2) are mutually excluding each other. In fact, they are not. The issue is that one should view the lattice spacing as resolution of measurements, and in quantum mechanics results of measurements depend on the details of the measuring procedure. Thus, observation (5.1) is like observing 'zitterbewegung' of a particle: if one would measure trajectory of a particle it would be not even continuous.

\footnotetext{
${ }^{5}$ Note that within a local field theory the time orientation of the trajectories can be ascribed only to large mass of a particle and a fast change of the orientation sample with temperature would imply strong dependence of the mass on the temperature. The branes construction is rooted in string theory and the time orientation is due to non-locality.
} 
Similarly, even if one finds a classical solution with a field which falls off, say, as a power of the distance $r$ one cannot expect observing this behavior on the lattice with the lattice spacing $a$ mach smaller than $r$. The zero-point fluctuations of the fields would distort drastically the classical solution which is no longer valid in fact.

There is no detailed understanding of the role of the resolution. It is clear, however, that one deals with quite a general phenomenon which can be called stabilization of classical solutions with high-frequency oscillating fields [20,18]. In case of QCD the phenomenon of the shrinking of classical solutions by zero-point fluctuations was observed and studied in detail in case of the topological fermionic modes which in the continuum-theory language are ascribed to the instantons.

Thus, the $1 / a^{4}$ behaviour of the action could well be the ultraviolet-sensitive image of the $1 / r^{4}$ behaviour in the classical limit produced by measurements with high resolution.

\section{Acknowledgments}

We are greatful M.N. Chernodub, M. D' Elia, A. Di Giacomo, F.V. Gubarev, A. Nakamura, H. Verschelde for valuable discussions.

\section{References}

[1] J. Greensite, The Confinement problem in lattice gauge theory, Prog. Part. Nucl. Phys. 51 (2003) 1, [arXiv:hep-lat/0301023].

[2] V.I. Zakharov, From confining fields on the lattice to higher dimensions in the continuum, Braz. J. Phys. 37 (2007) 165 [arXiv:hep-ph/0612342]; Dual string from lattice Yang-Mills theory, AIP Conf. Proc. 756 (2005) 182, [arXiv:hep-ph/0501011].

[3] A.S. Gorsky, V.I. Zakharov, A. R. Zhitnitsky, On Classification of QCD defects via holography, Phys. Rev. D79 (2009) 106003, arXiv:0902.1842 [hep-ph].

[4] A. Gorsky, V. Zakharov, Magnetic strings in Lattice QCD as Nonabelian Vortices, Phys. Rev. D77 (2008) 045017, [arXiv:0707.1284];

M.N. Chernodub, Atsushi Nakamura, V.I. Zakharov, Deconfinement phase transition in mirror of symmetries, arXiv:0904.0946[hep-ph].

[5] A. Gorsky, M. Shifman, A. Yung, Non-Abelian meissner effect in Yang-Mills theories at weak coupling, Phys. Rev. D71 (2005) 045010, [arXiv:hep-th/0412082].

[6] T. Sakai, Sh. Sugimoto, Low energy hadron physics in holographic QCD, Prog. Theor. Phys. 113 (2003) 843, [arXiv:hep-th/0412141].

[7] E. Witten, Anti-de Sitter Space, Thermal Phase Transition, And Confinement In Gauge Theories, Adv. Theor. Math. Phys. 2 (1998) 505, [arXiv:hep-th/9803131].

[8] O. Bergman, G. Lifschytz, Holographic U(1)(A) and String Creation, JHEP 0704 (2007) 043, [arXiv:hep-th/0612289].

[9] M.N. Chernodub, A. D’Alessandro, M. D’Elia, V.I. Zakharov, Thermal monopoles and selfdual dyons in the Quark-Gluon Plasma, arXiv:0909.5441 [hep-ph] .

[10] M.N. Chernodub, V.I. Zakharov, Magnetic component of Yang-Mills plasma Phys. Rev. Lett. 98 (2007) 082002, [arXiv:hep-ph/0611228]. 
[11] A. D'Alessandro, M. D'Elia, Magnetic monopoles in the high temperature phase of Yang-Mills theories, Nucl. Phys. B799 (2008) 241, e-Print: arXiv:0711.1266 [hep-lat]

A. D'Alessandro, M. D'Elia, Magnetic monopoles in high temperature QCD, PoS CONFINEMENT8 127, 2008. e-Print: arXiv:0812.1867 [hep-lat].

[12] M.N. Chernodub, F.V. Gubarev, M.I. Polikarpov, A. I. Veselov, Monopoles in the Abelian Projection of Gluodynamics , Progr. Theor. Phys. Suppl., 131. (1998) 309, [arXiv:hep-lat/9802036];

A. Di Giacomo, Monopole condensation and colour confinement, Progr. Theor. Phys. Suppl., 131 (1998) 161, [arXiv:hep-lat/9802008];

T. Suzuki, Low-energy effective theories from QCD, Progr. Theor. Phys. Suppl., 131 (1998) 633.

[13] A.M. Polyakov, ”Gauge Fields and Strings", Harvard Academic Publishers, (1987);

J. Ambjorn, "Quantization of geometry", [arXiv:hep-th/9411179].

[14] V.G. Bornyakov et al., Anatomy of the lattice magnetic monopoles, Phys. Lett, B537 (2002) 291, [arXiv:hep-lat/0103032].

[15] Sh. Ejiri, Sh. Kitahara, Y.Matsubara, T. Suzuki, String tension and monopoles in T not $=0$ SU(2) QCD, Phys. Lett. B343 (1995) 304, [arXiv:hep-lat/9407022];

S. Ejiri, Monopole condensation and quark confinement at finite temperature QCD, Nucl. Phys. A629 (1998) 89C.

[16] V.G. Bornyakov, P.Yu. Boyko, M.I. Polikarpov, V.I. Zakharov, Monopole clusters at short and large distances, Nucl. Phys. B672 (2003) 222, [arXiv:hep-lat/0305021].

[17] M.N. Chernodub, R. Feldmann, E.-M. Ilgenfritz, A. Schiller, Monopole chains in the compact Abelian Higgs model with doubly-charged matter field, Phys. Lett. B605 (2005) 161, e-Print: hep-lat/0406015 .

[18] A. Di Giacomo, V.I. Zakharov, Magnetic degrees of freedom in pure Yang-Mills theories, [arXiv:0806.2938 [hep-th]].

[19] R. C. Myers, Non-Abelian phenomena on D branes, Class. Quant. Grav. 20 (2003) S347, [arXiv:hep-th/0303072].

[20] V.I. Zakharov, Matter of resolution: From quasiclassics to fine tuning, published in Sense of Beauty in Physics, Miniconference in Honor of Adriano Di Giacomo on his 70th Birthday, Pisa, Italy, 26-27 Jan 2006, [arXiv:hep-ph/0602141].

[21] V.I. Zakharov, Shrinking fermionic modes, on the lattice and in the continuum, [arXiv:hep-ph/0612341]; $\mathrm{Ph}$. de Forcrand, Localization properties of fermions and bosons, AIP Conf.Proc. 892 (2007) 29, [arXiv:hep-lat/0611034]. 